\title{
Geometric realization of $\gamma$-vectors of subdivided cross polytopes
}

\author{
Natalie Aisbett \\ School of Mathematics and Statistics \\ University of Sydney \\ NSW, Australia \\ natalie.aisbett1@gmail.com
}

\author{
Vadim Volodin \\ Steklov Mathematical Institute \\ Moscow, Russia \\ volodinvadim@gmail.com
}

Submitted: Jan 18, 2020; Accepted: May 13, 2020; Published: Jun 12, 2020

(C) The authors. Released under the CC BY-ND license (International 4.0).

\begin{abstract}
For any flag simplicial complex $\Theta$ obtained by stellar subdividing the boundary of the cross polytope in edges, we define a flag simplicial complex $\Delta(\Theta)$ whose $f$ vector is the $\gamma$-vector of $\Theta$. This proves that the $\gamma$-vector of any such simplicial complex is the face vector of a flag simplicial complex, partially solving a conjecture by Nevo and Petersen. As a corollary we obtain that such simplicial complexes satisfy the Frankl-Füredi-Kalai inequalities.
\end{abstract}

Mathematics Subject Classifications: 05E45

\section{Introduction}

This paper relates to the theory of face enumeration of simplicial complexes. It gives a partial solution to a conjecture by Nevo and Petersen [13] on flag homology spheres, which are a particular class of simplicial complexes whose definition can be found in [4]. The conjecture is proven for a subclass of flag homology spheres, namely those that can be obtained by subdividing the boundary of the cross polytope in edges.

Recall that for a $(d-1)$-dimensional simplicial complex $\Theta$, the $f$-polynomial is a polynomial in $\mathbb{Z}[t]$ defined as follows:

$$
f(\Theta)(t):=f_{0}+f_{1} t+\cdots+f_{d} t^{d},
$$

where $f_{i}(\Theta)$ is the number of $(i-1)$-dimensional faces of $\Theta$, and $f_{0}(\Theta)=1$. The $h$ polynomial is given by

$$
h(\Theta)(t):=(1-t)^{d} f(\Theta)\left(\frac{t}{1-t}\right) .
$$


When $\Theta$ is a homology sphere $h(\Theta)$ is symmetric (this is known as the Dehn-Sommerville relations) hence it can be written

$$
h(\Theta)(t)=\sum_{i=0}^{\left\lfloor\frac{d}{2}\right\rfloor} \gamma_{i} t^{i}(1+t)^{d-2 i},
$$

for some $\gamma_{i} \in \mathbb{Z}$. Then the $\gamma$-polynomial is given by

$$
\gamma(\Theta)(t):=\gamma_{0}+\gamma_{1} t+\cdots+\gamma_{\left\lfloor\frac{d}{2}\right\rfloor} t^{\left\lfloor\frac{d}{2}\right\rfloor} .
$$

The vectors of coefficients of the $f$-polynomial, $h$-polynomial and $\gamma$-polynomial are known respectively as the $f$-vector, $h$-vector and $\gamma$-vector.

If $P$ is a simple $d$-dimensional polytope then the dual simplicial complex $\Theta_{P}$ of $P$ is the boundary complex (of dimension $d-1$ ) of the polytope that is polar dual to $P$. The dual simplicial complex of a $d$-dimensional cube is the boundary complex of the cross polytope and is denoted $\Sigma_{d-1}$. Shaving a codimension two face of a simple polytope is equivalent to the stellar subdivision in an edge of the dual simplicial complex. The set of simplicial complexes that can be obtained from $\Sigma_{d-1}$ by stellar subdivisions in edges is denoted $s d\left(\Sigma_{d-1}\right)$. By Corollary 5 the simplicial complexes in $s d\left(\Sigma_{d-1}\right)$ are flag homology spheres.

Recall that nestohedra are a broad class of simple polytopes introduced in [15] and [16]. Volodin [17] has shown that any $d$-dimensional flag nestohedron can be obtained from shaving codimension two faces of the $d$-dimensional cube. Hence, the set $s d\left(\Sigma_{d-1}\right)$ includes the dual simplicial complex to $d$-dimenisonal flag nestohedra.

Gal conjectured:

Conjecture 1. [10, Conjecture 2.1.7]. If $\Theta$ is a flag homology sphere then $\gamma(\Theta)$ is non negative.

Gal's conjecture was proved for all $\Theta$ in the class $s d\left(\Sigma_{d-1}\right)$ by Volodin in [17, Theorem 9]. This work was done with polytopes rather than their dual simplicial complexes. Further progress on Gal's conjecture was made in Athanasiadis in [4], where he studies subdivisions of $\Sigma_{d-1}$ of a more general kind.

Nevo and Petersen conjectured the following strengthening of Gal's conjecture:

Conjecture 2. [13, Problem 6.4]. If $\Theta$ is a flag homology sphere then $\gamma(\Theta)$ is the $f$ polynomial of a flag simplicial complex.

They proved this in [13] for the following classes of flag simplicial spheres:

- $\Theta$ is a Coxeter complex,

- $\Theta$ is the simplicial complex dual to an associahedron,

- $\Theta$ is the simplicial complex dual to a cyclohedron, 
- $\Theta$ has $\gamma_{1}(\Theta) \leqslant 3$,

This Conjecture is also supported by the more recent results of Labbé and Nevo in [12]. Aisbett has shown in [1] that this conjecture holds for the dual simplicial complex of all flag nestohedera. In this paper we prove Conjecture 2 for all $\Theta \in \operatorname{sd}\left(\Sigma_{d-1}\right)$ :

Theorem 3. Suppose that $\Theta \in s d\left(\Sigma_{d-1}\right)$. Then there is a flag simplicial complex $\Delta(\Theta)$ such that $f(\Delta(\Theta))=\gamma(\Theta)$.

The definition of $\Delta(\Theta)$ given in this paper is dependent upon the sequence of subdivisions of $\Sigma_{d-1}$ that produce $\Theta$. For dimensions 2 and 3 the required complex is a set of $\gamma_{1}(\Theta)$ points. For dimensions 4 and 5 the required complex is a graph with $\gamma_{1}(\Theta)$ vertices and $\gamma_{2}(\Theta)$ edges without triangles. This construction coincides with the simplicial complexes defined in [1] when $\Theta$ is the dual simplicial complex of a flag nestohedron, a proof of which can be found in [2]. This is not immediately obvious, since the definition in [1] used the language of building sets.

Frohmader [9, Theorem 1.1] showed that the $f$-vector of any flag simplicial complex satisfies the Frankl-Füredi-Kalai inequalities (see [8]). So Theorem 1.3 implies that $\gamma(\Theta)$ satisfies the Frankl-Füredi-Kalai inequalities, for all $\Theta \in s d\left(\Sigma_{d-1}\right)$. In [14] Nevo, Petersen and Tenner show that the $f$-vector of the barycentric subdivision of a homology sphere satisfies the Frankl-Füredi-Kalai inequalitites.

The main result of this paper was proven independently by Volodin in [19] and [18], and by Aisbett in [3]. The current paper is an amalgamation of the authors' results. The results and constructions discussed in this article formed the basis of the theory of a wide class of polyhedra, the theory of 2-truncated cubes; see $[5,6]$. This theory has been developed and found applications in the framework of Toric topology, a new direction of algebraic topology; see [7].

\section{Definitions}

A simplicial complex $\Theta$ with a finite vertex set $V_{\Theta}$ is a set of subsets of $V_{\Theta}$ such that

- $\{i\} \in \Theta$ for every $i \in V_{\Theta}$, and

- if $I \in \Theta$, and $J \subseteq I$, then $J \in \Theta$.

Elements of a simplicial complex are called faces, and the dimension of a face $F$ is equal to $|F|-1$. Faces of dimension 0 are called vertices, faces of dimension 1 are called edges, and a facet is a face that is not properly contained in any other face. The dimension of a simplicial complex is the maximal dimension of all its faces. The 1-dimensional simplicial complex that consists of the 0 and 1-dimensional faces of a simplicial complex $\Theta$ is called the 1-skeleton of $\Theta$. Observe that a 1-dimensional simplicial complex is also a graph, so we will use graph terminology when referring to 1-skeletons.

A simplicial complex is flag if for every set $S \subseteq V_{\Theta}$ with the property that $\{a, b\} \in \Theta$ for all $a, b \in S$, the set $S$ is a face of $\Theta$. A flag simplicial complex $\Theta$ is determined by 
its underlying 1-skeleton, since the faces of $\Theta$ are those subsets of vertices that form a complete graph.

Suppose $\Theta$ is a simplicial complex with vertices $V_{\Theta}$. If $W \subseteq V_{\Theta}$, then $\left.\Theta\right|_{W}$ denotes the restriction of $\Theta$ to $W$. The restriction $\left.\Theta\right|_{W}$ is the simplicial complex with vertex set $W$, such that $F$ is a face in $\left.\Theta\right|_{W}$ if and only if $F$ is a face in $\Theta$. A subcomplex of $\Theta$ is a simplicial complex whose vertex set is a subset of $V_{\Theta}$, and whose faces form a subset of the faces of $\Theta$.

The link of a face $F$ in a simplicial complex $\Theta$, denoted $\mathrm{lk}_{\Theta}(F)$, is the following subcomplex of $\Theta$ :

$$
\operatorname{lk}_{\Theta}(F):=\{G \in \Theta \mid G \cup F \in \Theta, G \cap F=\varnothing\} .
$$

If a simplicial complex $\Theta$ is flag, then $\operatorname{lk}_{\Theta}(F)$ is the restriction of $\Theta$ to the set of vertices in $V_{\Theta}-F$ that are adjacent to every vertex in $F$.

The stellar subdivision or subdivision of a simplicial complex $\Theta$ in the face $F$ is the simplicial complex $\Theta^{\prime}$ given by:

- $\Theta^{\prime}$ has vertices $V_{\Theta^{\prime}}=V_{\Theta} \cup\{s\}$ where $s \notin V_{\Theta}$,

- if $G \in \Theta$ and $F \nsubseteq G$, then $G \in \Theta^{\prime}$,

- if $H \in \Theta, F \nsubseteq H$, and $H \cup F \in \Theta$, then $H \cup\{s\} \in \Theta^{\prime}$.

If $F$ is simplex, we denote by $F^{\circ}$ the stellar subdivision of $F$ in the face $F$.

If $\Theta_{1}$ and $\Theta_{2}$ are simplicial complexes, then the join of $\Theta_{1}$ and $\Theta_{2}$, denoted $\Theta_{1} * \Theta_{2}$, is the simplicial complex on the vertex set $V_{\Theta_{1}} \cup V_{\Theta_{2}}$ defined by

$$
\Theta_{1} * \Theta_{2}:=\left\{F_{1} \cup F_{2} \mid F_{1} \in \Theta_{1}, F_{2} \in \Theta_{2}\right\} .
$$

Simplicial complexes $\Theta_{1}$ and $\Theta_{2}$ are equivalent, denoted $\Theta_{1} \cong \Theta_{2}$, if there is an inclusion preserving bijection between their faces.

Claim 4. (See [4, End of Section 2]). If $\Theta$ is a flag simplicial complex, and we perform a stellar subdivision on an edge $S$ of $\Theta$ to obtain the simplicial complex $\Theta^{\prime}$, then $\Theta^{\prime}$ is a flag simplicial complex.

Proof. Consider a set $L$ of vertices of $\Theta^{\prime}$ such that any pair of vertices in $L$ is an edge in $\Theta^{\prime}$. We will show that $L \in \Theta^{\prime}$.

If $s \notin L$ then every two element subset of $L$ does not include $s$, hence they were in $\Theta$. This implies that $L$ was in $\Theta$ and since $S \nsubseteq L$ this implies that $L \in \Theta^{\prime}$.

Suppose that $s \in L$. Let $H$ denote $L \backslash\{s\}$. Then all two element sets in $H$ are in $\Theta$ so that $H \in \Theta$. Since $\{s\} \cup\{v\} \in \Theta^{\prime}$ for all $v \in H$ this implies that $\{v\} \cup S \in \Theta$ for all $v \in H$. This implies that $H \cup S \in \Theta$ since $\Theta$ is flag, and hence that $L \in \Theta^{\prime}$.

Recall that $s d\left(\Sigma_{d-1}\right)$ denotes the set of simplicial complexes that can be obtained from $\Sigma_{d-1}$ by a sequence of edge subdivisions. 
Corollary 5. The simplicial complexes in $s d\left(\Sigma_{d-1}\right)$ are flag homology spheres.

Proof. The simplicial complexes in $s d\left(\Sigma_{d-1}\right)$ are flag by Claim 4 , and they are homology spheres since stellar subdivisions do not change the topology of the simplicial complex.

The following Proposition assists us in our purpose of proving the main result of this paper, Theorem 10. It describes how the $\gamma$-polynomial of a flag homology sphere changes under an edge subdivision.

Proposition 6. [10, Proposition 2.4.3]. Suppose $\Theta^{\prime}$ is a flag homology sphere obtained from a flag homology sphere $\Theta$ by stellar subdividing an edge $S$. Then

$$
\gamma\left(\Theta^{\prime}\right)-\gamma(\Theta)=t \gamma\left(\operatorname{lk}_{\Theta}(S)\right)
$$

Proof. If we stellar subdivide a face $F$ in a simplicial complex $\Theta$ to obtain $\Theta^{\prime}$, the change in the $f$-vector is

$$
f\left(\Theta^{\prime}\right)-f(\Theta)=f\left(F^{\circ} * \operatorname{lk}(F)\right)-f(F * \operatorname{lk}(F)),
$$

since the set of faces in $\Theta-\Theta^{\prime}$ is $F * \operatorname{lk}(F)$ and the set of faces in $\Theta^{\prime}-\Theta$ is $F^{\circ} * \operatorname{lk}(F)$. In general for simplicial complexes $A$ and $B$ we have

$$
f(A * B)=f(A) f(B) .
$$

Hence

$$
\begin{gathered}
f\left(\Theta^{\prime}\right)-f(\Theta)=f\left(\mathrm{lk}_{\Theta}(S)\right)\left[f\left(S^{\circ}\right)-f(S)\right] \\
=f\left(\mathrm{lk}_{\Theta}(S)\right)\left[1+3 t+2 t^{2}-\left(1+2 t+t^{2}\right)\right]=f\left(\operatorname{lk}_{\Theta}(S)\right)[t(1+t)] .
\end{gathered}
$$

If $\Theta$ is of dimension $(d-1)$, then

$$
\begin{gathered}
h\left(\Theta^{\prime}\right)-h(\Theta)=(1-t)^{d} f\left(\operatorname{lk}_{\Theta}(S)\right)\left(\frac{t}{1-t}\right)\left[\frac{t}{1-t}\left(1+\frac{t}{1-t}\right)\right] \\
=(1-t)^{d} f\left(\operatorname{lk}_{\Theta}(S)\right)\left(\frac{t}{1-t}\right)\left[\frac{t}{(1-t)^{2}}\right] \\
=t(1-t)^{d-2} f\left(\operatorname{lk}_{\Theta}(S)\right)\left(\frac{t}{1-t}\right) \\
=t \cdot h\left(\operatorname{lk}_{\Theta}(S)\right) .
\end{gathered}
$$

So

$$
\gamma\left(\Theta^{\prime}\right)\left(\frac{t}{(1+t)^{2}}\right)-\gamma(\Theta)\left(\frac{t}{(1+t)^{2}}\right)=\frac{t}{(1+t)^{2}} \gamma\left(\operatorname{lk}_{\Theta}(S)\right)\left(\frac{t}{(1+t)^{2}}\right) .
$$

The result follows. 


\section{Subdivision sequences}

For the purposes of this argument, say that a subdivision sequence is a sequence of simplicial complexes

$$
\left(\Theta^{0}, \Theta^{1}, \ldots, \Theta^{m}\right)
$$

where $\Theta^{0}$ is equivalent to $\Sigma_{d-1}$ for some $d$ and each $\Theta^{i}(i=1, \ldots, m)$ is obtained from $\Theta^{i-1}$ by subdividing an edge. (Not up to equivalence, but literally, so the set of vertices of $\Theta^{i}$ consists of the set of vertices of $\Theta^{i-1}$ together with one new vertex). For $i=1,2, \ldots, m$ we label the unique vertex of $\Theta^{i}$ that is not contained in $\Theta^{i-1}$ by $w_{i}$, so that $V_{\Theta^{i}}-V_{\Theta^{0}}=$ $\left\{w_{1}, w_{2}, \ldots, w_{i}\right\}$ for all $i$.

Suppose that $\left(\Theta^{0}, \Theta^{1}, \ldots, \Theta^{m}\right)$ is a subdivision sequence, and that we have subdivided $\Theta^{m-1}$ in the face $G=\left\{g_{a}, g_{b}\right\}$ to obtain $\Theta^{m}$. Define a set of vertices $V^{m}(F)$ (a subset of $\left.w_{1}, \ldots, w_{m}\right)$ for each face $F \in \Theta^{m}$ inductively as follows:

Let

$$
V^{m}(F)=\cap_{v \in F} V^{m}(\{v\}),
$$

and let

$$
\begin{aligned}
& V^{m}\left(\left\{w_{m}\right\}\right)=V^{m-1}(G), \\
& V^{m}(\{v\})=V^{m-1}(\{v\}) \cup\left\{w_{m}\right\} \quad \text { if }\left\{w_{m}\right\} \in \operatorname{lk}_{\Theta^{m}}(\{v\}) \text { and } v \notin\left\{g_{a}, g_{b}\right\}, \text { and } \\
& V^{m}(\{v\})=V^{m-1}(\{v\}), \quad \text { otherwise. }
\end{aligned}
$$

When no subdivisions are performed let $V^{0}(\{v\})=\varnothing$ for all $\{v\} \in \Sigma_{d-1}=\Theta^{0}$.

Define a flag simplicial complex $\Delta\left(\Theta^{m}\right)$ inductively as follows. Obtain the one-skeleton of $\Delta\left(\Theta^{m}\right)$ by attaching $w_{m}$ to the vertices in $V^{m-1}(G)$ of $\Delta\left(\Theta^{m-1}\right)$, and let $\Delta\left(\Theta^{0}\right)=\varnothing$. For all $m$, and for all $F \in \Theta^{m}$, let $\Delta^{m}(F)$ denote the flag simplicial complex $\left.\Delta\left(\Theta^{m}\right)\right|_{V^{m}(F)}$, so that $V^{m}(F)=V_{\Delta^{m}(F)}$.

Suppose $\left(\Theta^{0}, \ldots, \Theta^{m}\right)$ is a subdivision sequence, and that the face $G=\left\{g_{a}, g_{b}\right\}$ of $\Theta^{m-1}$ is subdivided to obtain $\Theta^{m}$. Observe that every $F \in \Theta^{m}$ lies in one of the following five sets:

$$
\begin{aligned}
& \mathcal{F}_{1}:=\left\{F \in \Theta^{m} \mid g_{a} \text { or } g_{b} \in F, \text { and } w_{m} \notin F\right\}, \\
& \mathcal{F}_{2}:=\left\{F \in \Theta^{m} \mid g_{a} \text { or } g_{b} \in F, \text { and } w_{m} \in F\right\}, \\
& \mathcal{F}_{3}:=\left\{F \in \Theta^{m} \mid g_{a}, g_{b} \notin F, \text { and } w_{m} \in F\right\}, \\
& \mathcal{F}_{4}:=\left\{F \in \Theta^{m} \mid g_{a}, g_{b}, w_{m} \notin F, \text { and }\left\{w_{m}\right\} \in \operatorname{lk}_{\Theta^{m}}(F)\right\}, \\
& \mathcal{F}_{5}:=\left\{F \in \Theta^{m} \mid g_{a}, g_{b}, w_{m} \notin F, \text { and }\left\{w_{m}\right\} \notin \operatorname{lk}_{\Theta^{m}}(F)\right\} .
\end{aligned}
$$

The following lemma, which describes how the vertex sets $V^{m}(F)$ change under an edge subdivision, is used to prove Proposition 8, which describes how the simplicial complexes $\Delta^{m}(F)$ change under edge subdivisions. For ease of notation we will write $V^{m}(v)$ rather than $V^{m}(\{v\})$ for vertices $v$.

Lemma 7. Suppose $\left(\Theta^{0}, \Theta^{1}, \ldots, \Theta^{m}\right)$ is a subdivision sequence, and that $\Theta^{m-1}$ is subdivided in the edge $G=\left\{g_{a}, g_{b}\right\}$ to obtain $\Theta^{m}$. Then 
if $F \in \mathcal{F}_{1}$, then

$$
V^{m}(F)=V^{m-1}(F)
$$

if $F \in \mathcal{F}_{2}$ and $g_{a} \in F$, then

$$
V^{m}(F)=V^{m-1}\left(F-\left\{w_{m}\right\} \cup\left\{g_{b}\right\}\right),
$$

and the same result is true with $g_{a}$ and $g_{b}$ swapped,

if $F \in \mathcal{F}_{3}$, then

$$
V^{m}(F)=V^{m-1}\left(F-\left\{w_{m}\right\} \cup G\right),
$$

if $F \in \mathcal{F}_{4}$, then

$$
V^{m}(F)=V^{m-1}(F) \cup\left\{w_{m}\right\},
$$

if $F \in \mathcal{F}_{5}$, then

$$
V^{m}(F)=V^{m-1}(F) .
$$

Proof. Suppose $F \in \mathcal{F}_{1}$. Without loss of generality we assume that $g_{a} \in F$. Then

$$
\begin{aligned}
V^{m}(F) & =\cap_{v \in F} V^{m}(v) \\
& =V^{m}\left(g_{a}\right) \cap_{v \in F \backslash\left\{g_{a}\right\}} V^{m}(v) \\
& =V^{m-1}\left(g_{a}\right) \cap_{v \in F \backslash\left\{g_{a}\right\}} V^{m-1}(v) \\
& =V^{m-1}(F) .
\end{aligned}
$$

The third equality follows from the fact that for any $v \in F \backslash\left\{g_{a}\right\}$ either $V^{m}(v)=$ $V^{m-1}(v)$ or $V^{m}(v)=V^{m-1}(v) \cup\left\{w_{m}\right\}$, and $w_{m} \notin V^{m}\left(g_{a}\right)$.

Suppose that $F \in \mathcal{F}_{2}$, and without loss of generality that $g_{a} \in F$. Then

$$
\begin{aligned}
V^{m}(F) & =\cap_{v \in F} V^{m}(v) \\
& =V^{m}\left(g_{a}\right) \cap V^{m}\left(w_{m}\right) \cap_{v \in F \backslash\left\{g_{a}, w_{m}\right\}} V^{m}(v) \\
& =V^{m-1}\left(g_{a}\right) \cap V^{m-1}\left(g_{b}\right) \cap_{v \in F \backslash\left\{g_{a}, w_{m}\right\}} V^{m-1}(v) \\
& =V^{m-1}\left(F-\left\{w_{m}\right\} \cup\left\{g_{b}\right\}\right) .
\end{aligned}
$$

The third equality follows from the fact that $V^{m}\left(w_{m}\right)=V^{m-1}\left(g_{a}\right) \cap V^{m-1}\left(g_{b}\right)$, and the fact that for all $v \in F \backslash\left\{g_{a}, w_{m}\right\}, V^{m}(v)=V^{m-1}(v) \cup\left\{w_{m}\right\}$ however $w_{m} \notin V^{m}\left(g_{a}\right)$.

Suppose that $F \in \mathcal{F}_{3}$. Then

$$
\begin{aligned}
V^{m}(F) & =\cap_{v \in F} V^{m}(v) \\
& =V^{m}\left(w_{m}\right) \cap_{v \in F \backslash\left\{w_{m}\right\}} V^{m}(v) \\
& =V^{m-1}\left(g_{a}\right) \cap V^{m-1}\left(g_{b}\right) \cap_{v \in F \backslash\left\{w_{m}\right\}} V^{m-1}(v) \\
& =V^{m-1}\left(F-\left\{w_{m}\right\} \cup G\right) .
\end{aligned}
$$

The third equality follows from the fact that $V^{m}\left(w_{m}\right)=V^{m-1}\left(g_{a}\right) \cap V^{m-1}\left(g_{b}\right)$, and the fact that for all $v \in F \backslash\left\{w_{m}\right\}, V^{m}(v)=V^{m-1}(v) \cup\left\{w_{m}\right\}$ however $w_{m} \notin V^{m}\left(w_{m}\right)$. 
Suppose that $F \in \mathcal{F}_{4}$. Then

$$
\begin{aligned}
V^{m}(F) & =\cap_{v \in F} V^{m}(v) \\
& =\cap_{v \in F}\left(V^{m-1}(v) \cup\left\{w_{m}\right\}\right) \\
& =V^{m-1}(F) \cup\left\{w_{m}\right\} .
\end{aligned}
$$

Suppose that $F \in \mathcal{F}_{5}$. Then

$$
\begin{aligned}
V^{m}(F) & =\cap_{v \in F} V^{m}(v) \\
& =\cap_{v \in F} V^{m-1}(v) \\
& =V^{m-1}(F) .
\end{aligned}
$$

The second equality follows from the fact that for all $v \in F$ either $V^{m}(v)=V^{m-1}(v)$ or $V^{m}(v)=V^{m-1}(v) \cup\left\{w_{m}\right\}$. Since not every vertex in $F$ is adjacent to $w_{m}$, this implies that for some $v \in F$, we have $V^{m}(v)=V^{m-1}(v)$.

Proposition 8. Suppose $\left(\Theta^{0}, \Theta^{1}, \ldots, \Theta^{m}\right)$ is a subdivision sequence. Then

if $F \in \mathcal{F}_{1}$ then

$$
\Delta^{m}(F)=\Delta^{m-1}(F),
$$

if $F \in \mathcal{F}_{2}$ and $g_{a} \in F$, then

$$
\Delta^{m}(F)=\Delta^{m-1}\left(F-\left\{w_{m}\right\} \cup\left\{g_{b}\right\}\right),
$$

and the same result holds with $g_{a}$ and $g_{b}$ swapped,

if $F \in \mathcal{F}_{3}$ then

$$
\Delta^{m}(F)=\Delta^{m-1}\left(F-\left\{w_{m}\right\} \cup G\right),
$$

if $F \in \mathcal{F}_{4}$ then

$$
\Delta^{m}(F)=\Delta^{m-1}(F) \cup \Delta^{m-1}(F \cup G) *\left\{w_{m}\right\},
$$

if $F \in \mathcal{F}_{5}$ then

$$
\Delta^{m}(F)=\Delta^{m-1}(F)
$$

Proof. Suppose that $F \in \mathcal{F}_{1}$. Then since $V^{m}(F)=V^{m-1}(F)$, and $\Delta\left(\Theta^{m}\right)$ is obtained from $\Delta\left(\Theta^{m-1}\right)$ by adding faces that contain $w_{m}$ only, it follows immediately that $\Delta^{m}(F)=$ $\Delta^{m-1}(F)$.

Suppose that $F \in \mathcal{F}_{2}$. Then $V^{m}(F)=V^{m-1}\left(F-\left\{w_{m}\right\} \cup\left\{g_{b}\right\}\right)$ which is a subset of the vertices of $\Delta\left(\Theta^{m-1}\right)$, hence it follows immediately that $\Delta^{m}(F)=\Delta^{m-1}\left(F-\left\{w_{m}\right\} \cup\left\{g_{b}\right\}\right)$. 
Suppose that $F \in \mathcal{F}_{3}$. Then since $V^{m}(F)=V^{m-1}\left(F-\left\{w_{m}\right\} \cup G\right)$ it follows immediately that $\Delta^{m}(F)=\Delta^{m-1}\left(F-\left\{w_{m}\right\} \cup G\right)$.

Suppose that $F \in \mathcal{F}_{4}$. Then $V^{m-1}(F) \cap V^{m-1}(G)=\cap_{v \in F} V^{m-1}(v) \cap V^{m-1}\left(g_{a}\right) \cap$ $V^{m-1}\left(g_{b}\right)=V^{m-1}(F \cup G)$. Now $V^{m}(F)=V^{m-1}(F) \cup\left\{w_{m}\right\}$, and since $w_{m}$ is adjacent to the vertices $V^{m-1}(G)$ in $\Delta\left(\Theta^{m}\right)$ this implies $w_{m}$ is adjacent to the vertices $V^{m-1}(F \cup G)$ within $V^{m-1}(F)$. Therefore $\Delta^{m}(F)=\Delta^{m-1}(F) \cup \Delta^{m-1}(F \cup G) *\left\{w_{m}\right\}$.

Suppose that $F \in \mathcal{F}_{5}$. Then since $V^{m}(F)=V^{m-1}(F)$ it follows immediately that $\Delta^{m}(F)=\Delta^{m-1}(F)$.

\section{The main theorem}

In this section, we will prove the main result of this paper, Theorem 10 . We first prove a seemingly more general form of this theorem, Theorem 9. It shows that if $\left(\Theta^{0}, \ldots, \Theta^{m}\right)$ is a subdivision sequence, then the Nevo and Petersen Conjecture holds for the link of all faces in $\Theta^{m}$. However, for all faces $F \in \Theta^{m}$, it is true that $\operatorname{lk}_{\Theta^{m}}(F) \in \operatorname{sd}\left(\Sigma_{d^{\prime}}\right)$ for some $d^{\prime} \leqslant d-1$, so this theorem is not a generalisation of Theorem 10 .

Theorem 9. Suppose that $F \in \Theta^{m}$. Then $f\left(\Delta^{m}(F)\right)=\gamma\left(\operatorname{lk}_{\Theta^{m}}(F)\right)$.

Proof. We suppose that this holds by induction for all $k<m$. When $m=0$, for all faces $F \in \Theta^{0}=\Sigma_{d-1}$ we have that

$$
f\left(\Delta^{0}(F)\right)=1
$$

and

$$
\gamma\left(\operatorname{lk}_{\Theta^{0}}(F)\right)=1
$$

Suppose that $F \in \Theta^{m}$.

If $F \in \mathcal{F}_{1}$ then $\Delta^{m}(F)=\Delta^{m-1}(F)$ and $\operatorname{lk}_{\Theta^{m}}(F) \cong \mathrm{lk}_{\Theta^{m-1}}(F)$, so that

$$
f\left(\Delta^{m}(F)\right)=f\left(\Delta^{m-1}(F)\right)=\gamma\left(\operatorname{lk}_{\Theta^{m-1}}(F)\right)=\gamma\left(\operatorname{lk}_{\Theta^{m}}(F)\right)
$$

Suppose that $F \in \mathcal{F}_{2}$. Then $\Delta^{m}(F)=\Delta^{m-1}\left(F-\left\{w_{m}\right\} \cup\left\{g_{b}\right\}\right)$, and $\operatorname{lk}_{\Theta^{m}}(F) \cong \operatorname{lk}_{\Theta^{m-1}}(F-$ $\left.\left\{w_{m}\right\} \cup\left\{g_{b}\right\}\right)$, so that

$$
f\left(\Delta^{m}(F)\right)=f\left(\Delta^{m-1}\left(F-\left\{w_{m}\right\} \cup\left\{g_{b}\right\}\right)\right)=\gamma\left(\operatorname{lk}_{\Theta^{m-1}}\left(F-\left\{w_{m}\right\} \cup\left\{g_{b}\right\}\right)\right)=\gamma\left(\operatorname{lk}_{\Theta^{m}}(F)\right) .
$$

Suppose that $F \in \mathcal{F}_{3}$. Then $\Delta^{m}(F)=\Delta^{m-1}\left(F-\left\{w_{m}\right\} \cup G\right)$, and $\operatorname{lk}_{\Theta^{m}}(F) \cong \operatorname{lk}_{\Theta^{m-1}}(F-$ $\left.\left\{w_{m}\right\} \cup G\right) * \Sigma_{0}$, where $\Sigma_{0}$ is the complex with two non-adjacent vertices $g_{a}, g_{b}$. Therefore, 


$$
\begin{aligned}
f\left(\Delta^{m}(F)\right) & =f\left(\Delta^{m-1}\left(F-\left\{w_{m}\right\} \cup G\right)\right) \\
& =\gamma\left(\operatorname{lk}_{\Theta^{m-1}}\left(F-\left\{w_{m}\right\} \cup G\right)\right) \\
& =\gamma\left(\operatorname{lk}_{\Theta^{m-1}}\left(F-\left\{w_{m}\right\} \cup G\right)\right) \gamma\left(\Sigma_{0}\right) \\
& =\gamma\left(\operatorname{lk}_{\Theta^{m-1}}\left(\left(F-\left\{w_{m}\right\} \cup G\right) * \Sigma_{0}\right)\right. \\
& =\gamma\left(\operatorname{lk}_{\Theta^{m}}(F)\right) .
\end{aligned}
$$

Suppose $F \in \mathcal{F}_{4}$. Then $\Delta^{m}(F)=\Delta^{m-1}(F) \cup \Delta^{m}(F \cup G) *\left\{w_{m}\right\}$, and $\mathrm{lk}_{\Theta^{m}}(F)$ is obtained by subdividing $\operatorname{lk}_{\Theta^{m-1}}(F)$ in the edge $G$. Therefore, by Proposition 6

$$
f\left(\Delta^{m}(F)\right)=f\left(\Delta^{m-1}(F)\right)+t f\left(\Delta^{m-1}(F \cup G)\right)
$$

and

$$
\begin{aligned}
\gamma\left(\operatorname{lk}_{\Theta^{m}}(F)\right) & =\gamma\left(\operatorname{lk}_{\Theta^{m-1}}(F)\right)+t \gamma\left(\operatorname{lk}_{\operatorname{lk}_{\Theta^{m-1}}(F)}(G)\right) \\
& =\gamma\left(\operatorname{lk}_{\Theta^{m-1}}(F)\right)+t \gamma\left(\operatorname{lk}_{\Theta^{m-1}}(F \cup G)\right)
\end{aligned}
$$

and since $f\left(\Delta^{m-1}(F)\right)=\gamma\left(\operatorname{lk}_{\Theta^{m-1}}(F)\right)$ and $f\left(\Delta^{m-1}(F \cup G)\right)=\gamma\left(\operatorname{lk}_{\Theta^{m-1}}(F \cup G)\right)$ this implies that

$$
f\left(\Delta^{m}(F)\right)=\gamma\left(\operatorname{lk}_{\Theta^{m}}(F)\right)
$$

Suppose $F \in \mathcal{F}_{5}$. Then $\Delta^{m}(F)=\Delta^{m-1}(F)$, and $\operatorname{lk}_{\Theta^{m}}(F)=\mathrm{lk}_{\Theta^{m-1}}(F)$, so that

$$
f\left(\Delta^{m}(F)\right)=f\left(\Delta^{m-1}(F)\right)=\gamma\left(\operatorname{lk}_{\Theta^{m-1}}(F)\right)=\gamma\left(\operatorname{lk}_{\Theta^{m}}(F)\right) .
$$

Note that the empty face $\varnothing \in \Theta^{m}$ has link $\Theta^{m}$, i.e. $\operatorname{lk}_{\Theta^{m}}(\varnothing)=\Theta^{m}$. Observe also that $\varnothing \in \mathcal{F}_{4}$ for all $m$, and $V^{m}(\varnothing)=\left\{w_{1}, \ldots, w_{m}\right\}$, so that $\Delta^{m}(\varnothing)=\Delta\left(\Theta^{m}\right)$. This, along with Theorem 9 immediately implies the main result of this paper:

Theorem 10.

$$
f\left(\Delta\left(\Theta^{m}\right)\right)=\gamma\left(\Theta^{m}\right)
$$

Theorem 11 ([8, Frankl-Füredi-Kalai]). Denote by $\left(\begin{array}{l}n \\ k\end{array}\right)_{r}$ the number of $k$-cliques in the Turan graph $T_{n, r}$. For natural numbers $m, k$ and $r \geqslant k$ there exists a unique canonical representation

$$
m=\left(\begin{array}{c}
n_{k} \\
k
\end{array}\right)_{r}+\cdots+\left(\begin{array}{c}
n_{k-s} \\
k-s
\end{array}\right)_{r-s},
$$

where $n_{k-1}-\left\lfloor\frac{n_{k-i}}{r-i}\right\rfloor$ for all $0 \leqslant i<s$ and $n_{k-s} \geqslant k-s>0$. Define

$$
m^{\langle k\rangle_{r}}=\left(\begin{array}{c}
n_{k} \\
k+1
\end{array}\right)_{r}+\cdots+\left(\begin{array}{c}
n_{k-s} \\
k-s+1
\end{array}\right)_{r-s} .
$$

The integer vector $\left(f_{0}, \ldots, f_{n}\right)$ with non-negative components is the $f$-vector of some $r$ colorable simplicial complex $K$ if and only if $f_{k} \leqslant f_{k-1}^{\langle k\rangle_{r}}$. 
Then, using the Frankl-Füredi-Kalai inequalities we obtain the following result:

Corollary 12. Let $\Theta \in \operatorname{sd}\left(\Sigma_{d-1}\right)$. Then $0 \leqslant \gamma_{i} \leqslant \gamma_{k}^{\langle k\rangle_{r}}$, where $k>1, r=\left\lfloor\frac{n}{2}\right\rfloor$.

Let us apply the obtained result to polytopes of dimensions 4 and 5 . Their $\gamma$-vectors have only 3 components: $\left(1, \gamma_{1}, \gamma_{2}\right)$. In this case we obtain a graph with $\gamma_{1}$ vertice and $\gamma_{2}$ edges without triangles. Therefore, we have 3 inequalities:

1. $\gamma_{1} \geqslant 0$

2. $\gamma_{2} \geqslant 0$

3. $\gamma_{2} \leqslant \frac{\gamma_{1}\left(\gamma_{1}-1\right)}{2}$.

\section{Acknowledgements}

Volodin would like to thank his supervisor Victor Buchstaber for his attention to his work and useful discussions. This paper formed part of Aisbett's PhD research in the School of Mathematics and Statistics at the University of Sydney. She would like to thank her supervisor Anthony Henderson for his feedback and careful reading.

\section{References}

[1] N. Aisbett, Frankl-Füredi-Kalai Inequalities on the $\gamma$-Vectors of Flag Nestohedra, Discrete \& Computational Geometry, Vol. 51, 2014, pp. 323 - 336.

[2] N. Aisbett The Gamma-Polynomial of Flag Homology Spheres, PhD thesis, The University of Sydney, 2013 (unpublished).

[3] N. Aisbett, Gamma-vectors of edge subdivisions of the boundary of the cross polytope, arXiv: 1209.1789v1, 2012.

[4] C. A. Athanasiadis, Flag subdivisions and $\gamma$-vectors, Pacific J. Math. Vol.259, 2012, no. 2, pp. 257-278.

[5] V. M. Buchstaber, V. D. Volodin, Sharp upper and lower bounds for nestohedra., Izv. Math., 75:6, 2011, 1107-1133.

[6] V. M. Buchstaber, V. D. Volodin, Combinatorial 2-truncated cubes and applications, Associahedra, Tamari Lattices, and Related Structures, Tamari Memorial Festschrift, Progress in Mathematics, v. 299, Birkhäuser, Basel, 2012, 161-186.

[7] V. M. Buchstaber, T. E. Panov, Toric Topology, Mathematical Surveys and Monographs, v. 204, Amer. Math. Soc., Providence, RI, 2015, 518 pp

[8] P. Frankl, Z. Füredi, G. Kalai, Shadows of colored complexes, Math. Scand. Vol. 63, 1988, pp. 169-178.

[9] A. Frohmader, Face vectors of flag complexes, Israel J. Math, Vol. 164, 2008, pp. 153-164. 
[10] S. R. Gal, Real Root Conjecture Fails for Five-and-Higher Dimensional Spheres, Discrete Comput. Geom, Vol. 34, 2005, no. 2, pp. 269-284.

[11] V. Klee, A combinatorial analogue of Poincaré's duality theorem, Can. J. Math. Vol. 16, 1964, pp. 517-531.

[12] J. P. Labbé, E. Nevo, Bounds for Entries of $\gamma$-Vectors of Flag Homology Spheres, SIAM Journal on Discrete Mathematics, Vol. 31, 2017, no. 3, pp. 2064-2078.

[13] E. Nevo, T. K. Petersen, On $\gamma$-Vectors Satisfying the Kruskal-Katona Inequalities, Discrete Comput. Geom, Vol. 45, 2010, pp. 503-521.

[14] E. Nevo, T. K. Petersen, B. E. Tenner, The $\gamma$-vector of a barycentric subdivision, J. Combin. Theory Ser. A, Vol. 118, 2011, pp. 1364-1380.

[15] A. Postnikov, Permutohedra, Associahedra and Beyond, International Mathematics Research Notices, 2009, no. 6, pp. 1026-1106.

[16] A. Postnikov, V. Reiner and L. Williams, Faces of Generalized Permutohedra, Doc. Math, Vol. 13, 2008, pp. 207-273.

[17] V. D. Volodin, Cubical realizations of flag nestohedra and a proof of Gal's Conjecture for them, Uspekhi Mat. Nauk, Vol. 65, 2010, no. 1, pp. 188-190.

[18] V. D. Volodin, Geometric realization of the $\gamma$-vectors of 2-truncated cubes, 2012, arXiv:210.0398v1.

[19] V. D. Volodin, Geometric realization of the $\gamma$-vectors of 2-truncated cubes, Russian Mathematical Surveys, Vol. 67, 2012, no. 3, pp. 582-584. 